\title{
Scrutinizing Unsolicited E-Mail And Revealing Zombies
}

\author{
${ }^{1}$ V.Annie, ${ }^{2}$ Mr. G. Sathishkumar. B.E, M.E, Ph.D.* \\ ${ }^{1}$ II YEAR M.E (CSE) Department of computer science \& engineering , ${ }^{2}$ RESEARCH SCHOLAR Department of \\ computer science \& engineering Mount Zion college of Engineering \& technology Sathiyabama University \\ Pudukkottai Chennai
}

\begin{abstract}
In this paper specialize in the detection of the compromised machines during a network that are concerned within the spamming activities. In this paper, considering the matter in unsought spam e-mail's wherever crucial information's are divided to assure data confidentiality and integrity. The replicas of divided shares are dynamically allotted to boost recital. Heuristic search minimizes the spam access in Zombies. During this paper, Network official examines unsought e-mail by mistreatment the heuristic search, and reportable to the tip user. Once checking the activities beside IP address, the unsought e-mail is blocked that are originated by Zombies.
\end{abstract}

Index term-Zombies, spam, secure information, secret sharing, and heuristic.

\section{Introduction}

In recent years, malware has become a widespread downside. Compromised machines on the web are typically stated as bots, and the set of bots controlled by one entity is termed a botnet. Botnet controllers use techniques like IRC channels and customized peer-to-peer protocols to manage and operate these bots. Botnets have multiple wicked uses: mounting DDoS attacks, stealing user passwords and identities, generating click fraud [5], and causing spam email [11]. there's anecdotal proof that spam may be a propulsion within the economic science of botnets: a standared strategy for monetizing botnets is causing spam email, wherever spam is outlined generously to incorporate ancient publicity email messages, likewise as phishing email messages, email messages with viruses, and different unwanted email messages.

During this paper, considering the matter in unsought spam e-mail's wherever crucial information's are divided to assure data confidentiality and integrity. The replicas of divided shares are dynamically allotted to boost recital. Heuristic search minimizes the spam access in zombies. It is thought-about that the work enhances in such the way that one specialise in the performance problems and also the different specialise in the protection assurance problems.

\section{Related Work}

During this section, tend to gift AutoRE - a framework for mechanically generating address signatures to spot Zombies-based spam campaigns. As input, AutoRE takes solely a collection of untagged email messages (messages aren't labeled as spam/non-spam), and professional duces 2 outputs: a collection of spam address signatures, and a connected list of Zombies host IP addresses.

Zombies, or additional properly unsought industrial E-mail (UCE), ar Associate in Nursing increasing threat to the viability of net E-mail and a danger to net commerce. UCE senders remove resources from users and repair suppliers while not compensation and while not authorization. a range of counter-measures to UCE are projected, from technical to regulative.

Among the technical ones, the utilization of filtering strategies is in style and effective. UCE filtering may be a text categorization task. Text categorization (TC) is that the classification of documents with relation to a collection of 1 or additional pre-existing classes. within the case of UCE, the task is to classify e-mail messages or newsgroups articles as UCE or not (that is, legitimate). The final model of TC makes use of a collection of pre-classified documents to classify new ones, per the text content (i.e. words) of the documents

Heuristic search take into account a secure information storage system that survives though some nodes within the system are compromised. It assumes that information are secret shared and also the full set of shares are replicated and statically distributed over the network. the foremost focus of this work is to ensure confidentiality and integrity necessities of the storage system. During this paper, take into account the message partitioning and replication to support secure, survivable, and high performance storage systems. Our goal is to dam the larva herder activities.

\section{Overview}

Here initial study the standard of the extracted address signatures. Here used the human classified labels to cipher the spam detection false positive rate. to raised perceive the effectiveness of mistreatment 
signatures for future spam detection, we tend to performed cross-month analysis by applying signatures generated during a previous month to emails received during a later month. These experiments conjointly incontestible the importance of getting regular expression signatures.

Second, Here examined whether or not the known Zombie hosts were so spamming servers - to the present finish, we tend to used the Hotmail server log that records the causing history of all email servers that communicate with Hotmail over time. This log includes the e-mail volume and also the spam magnitude relation four of every server on a usual. during this paper, these statistics to guage the known Zombie hosts.

The spam magnitude relation was computed mistreatment the present spam filtering system organized. this filter leverages each email content and email server causing history for spam detection. Finally, they're inquisitive about finding whether or not every set of emails known from constant spam campaign were properly classified along. To answer this question, for each set, we tend to examine the similarity between the corresponding destination sites. during this paper destination sites were shown to be powerfully related to to the corresponding spam campaign.

\section{Technique Utilized By Zombie}

Zombie/Spammers use numerous techniques to send massive volumes of mail whereas making an attempt to stay untraceable. Here describe many of those techniques, starting with .conventional. strategies and getting to additional tangled techniques.

\subsection{Direct spamming.}

Spammers could purchase upstream property from .spam-friendly ISPs., that flip a blind eye to the activity. sometimes, spammers purchase property and send spam from ISPs that don't forgive this activity and ar forced to alter ISPs. Ordinarily, dynamic from one ISP to a different would need a sender to renumber the IP addresses of their mail relays IP address spoofed to look as if it came from the dialup affiliation, and proxy the reverse traffic through the dialup affiliation back to the spamming hosts .

\subsection{Open relays and proxies.}

Open relays ar mail servers that enable unauthenticated net hosts to attach and relay email through them. Originally meant for user convenience (e.g., to let users send mail from a selected relay whereas they're traveling or otherwise during a totally different network), It seems that the widespread readying and use of blacklisting techniques have just about destroyed the utilization of open relays and proxies to send spam .

\subsection{Zombie/bot.}

Standard knowledge suggests that the bulk of spam on the web these days is shipped by Zombie. Collections of machines acting underneath one centralized controller . The W32/Bobax (.Bobax.) worm (of that there ar several variants) exploits the DCOM and LSASS vulnerabilities on Windows systems, permits infected hosts to be used as a mail relay, and makes an attempt to unfold itself to different machines plagued by the on top of vulnerabilities, likewise as over email. This studies the network level properties of spam sent by Bobax drones. Agobot and SDBot ar 2 different bots presupposed to send spam.
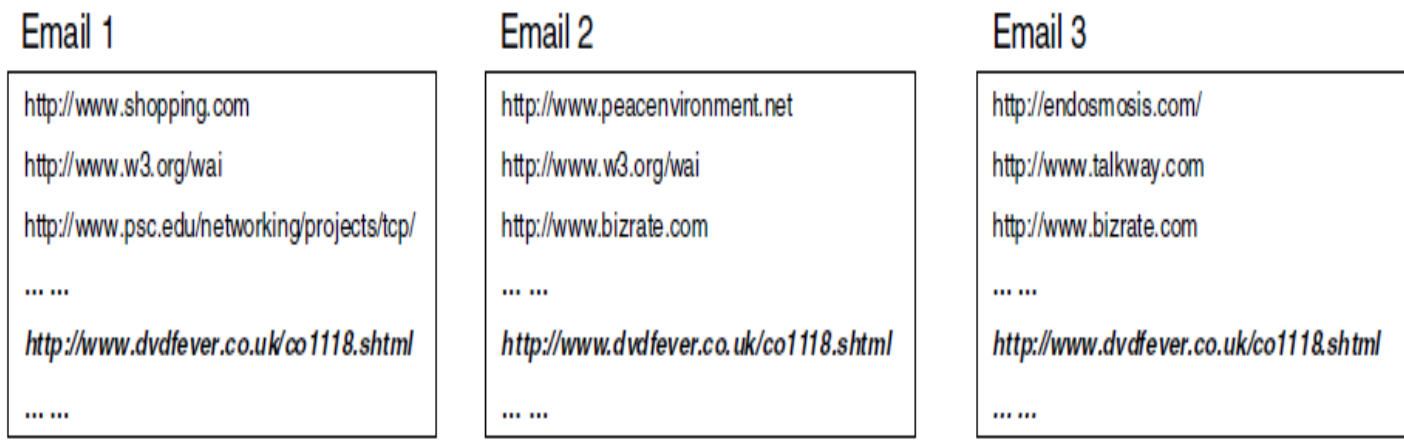

Figure 4.1: Multi-URL spam emails that we tend to suspect were sent from constant botnet. These emails were from totally different IP addresses, however were sent nearly at the same time.

\subsection{Spam from Dynamic IP Addresses}

The dynamic email servers sent emails to Hotmail one time throughout the course of 3 
month, the mass volume of spam from these servers remains massive

\begin{tabular}{|c|c|c|c|c|c|}
\hline & Total num. of IPs & $\begin{array}{c}\text { Num. of IPs used by } \\
\text { mail servers }\end{array}$ & $\begin{array}{c}\text { \% of emails } \\
\text { classified as spam }\end{array}$ & $\begin{array}{c}\text { \% of all Hotmail } \\
\text { incoming spam }\end{array}$ & $\begin{array}{c}\text { \% of user-reported } \\
\text { spam }\end{array}$ \\
\hline UDmap IP & $102,941,051$ & $24,115,951$ & $92.4 \%$ & $42.2 \%$ & $40.3 \%$ \\
\hline Dynablock IP & $193,808,955$ & $15,773,646$ & $92.3 \%$ & $30.4 \%$ & $29.3 \%$ \\
\hline UDmap IPI|Dynablock IP & $242,248,012$ & $27,163,219$ & $92.2 \%$ & $50.7 \%$ & $49.2 \%$ \\
\end{tabular}

This table shows that regarding ninety two of the emails from totally different IPs(UDmapIP ,dynamapIPs )are spam, accounting for up to fifty.7\% The importance of Associate in Nursing automatic technique for keeping track of most up to date, popularly used dynamic IPs.Given the high proportion of spam from dynamic IP addresses, a question we tend to raise is whether or not spam originates from simply a number of hosts. Based on the classification results mistreatment the existing Hotmail spam filter, 95.6\% of the sessions from UDmap IPs sent spam solely (spam magnitude relation $=100 \%$ ), $97.0 \%$ of them send emails with over ninetieth spam magnitude relation. The remaining third will doubtless be legitimate mail servers. we tend to note here, however, the three is Associate in Nursing boundary of our sender detection false positive rate as a result of the present spam filter may miss out spam emails. there's a way smaller fraction of sessions from the doubtless static IP addresses with a high spam ratio: thirty one.4\% of the sessions sent solely spam, and $62.8 \%$ of the sessions had spam magnitude relation less than ninetieth. Mistreatment the data of dynamic IP addresses, additional cut back the spam filtering false negatives that are misclassified by the present spam filter, however expressly reportable by users as spam.

\section{Spam Zombie Revealing Algorithms}

SPOT is intended supported the applied math tool SPRT we tend to mentioned within the last section. within the context of sleuthing spam zombies in SPOT, we tend to take into account H1 as a detection and H0 as a normality that within the context of spam zombie detection, from the point of view of network observation, it's additional necessary to spot the machines that are compromised than the machines that are traditional.

Once a machine is known as being compromised it's added into the list of doubtless compromised machines that system directors will follow to scrub. The message-sending behavior of the machine is additionally recorded ought to additional analysis be needed.

Before the machine is cleansed and far from the list, the SPOT detection system doesn't got to additional monitor the message causing behavior of the machine. On the opposite hand, a machine that's presently traditional could get compromised at a later time. Thus got to endlessly monitor machines that are determined to be traditional by SPOT. Once such a machine is known by SPOT, the records of the machine in SPOT are reset, particularly; observation section starts for the machine.

\subsection{Heuristic Algorithm:}

\section{Projected Algorithm:}

The options that are rare in traditional messages however seem ofttimes in spam, like non- existing domain names and spam-related keywords, is wont to distinguish spam from traditional email. Spam Assassin is such Associate in Nursing example. every received message is verified against the heuristic filtering rules. Compared with a pre- outlined threshold, the verification result decides whether or not the message is spam or not.

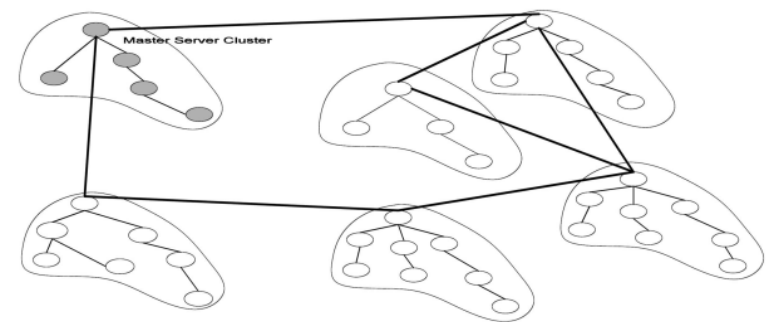

Figure 6.1:A sample graph of a system 


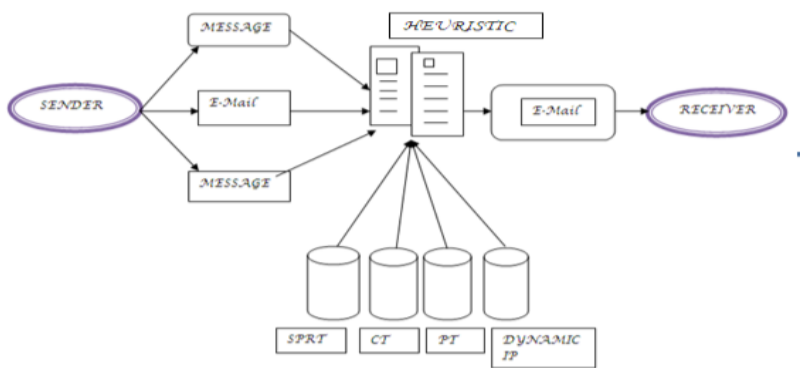

Figure 6.2:Heuristic algorithm

\subsection{Heuristics for ZOMBIE:}

Within the algorithmic program, Here projected a collection of heuristic options to enrich the word Bayesian model in their work, including: a collection of around thirty five hand stitched key phrases (like "free money"); some non text options (like the domain of the sender, or whether or not the message comes from a listing or not); and options regarding the non alphamerical characters within the messages.

For this work, we've targeted during this last set of options. The take a look at assortment employed in our experiments, Spam base, already contained a collection of 9 heuristic options. Spam base is Associate in Nursing e-mail messages assortment containing 4601 messages, being 1813 (39\%) marked as ZOMBIE. the gathering comes in preprocessed (not raw) kind, and its instances are diagrammatic as 58-dimensional vectors. the primary forty eight options ar words extracted from the initial messages, while not stop list nor stemming, and chosen because the most unbalanced words for the ZOMBIE category. consecutive six options are the share of occurrences of the special characters ";', "(", "[", "!", "\$" and "\#". the subsequent three options represent totally different measures of occurrences of capital letters within the text of the messages.

Finally, the last feature is that the category label. So, options forty nine to fifty seven represent heuristic attributes of the messages. In our experiments, we've tested many learning algorithms on 3 feature sets: just one This assortment is words, solely heuristic attributes, and both. we've divided the Spam base assortment in 2 parts: a ninetieth of the instances axe used for coaching, and a tenth of the messages are preserved for testing. This split has been performed protective the odds of legitimate and ZOMBIE messages within the whole assortment.

\section{Performance Evaluation:}

So as to grasp the performance of HEURISTIC in terms of the false positive and false negative rates, we tend to suppose variety of how to verify if a machine is so compromised. First, we tend to check if any say we've a confirmation. Out of the 132 IP addresses known by HEURISTIC, we are able to make sure a hundred and ten of them to be compromised during this means. For the remaining twenty two IP addresses, we tend to manually examine the spam causing patterns from the IP addresses and also the domain names of the corresponding machines. If the fraction of the spam messages from Associate in Nursing IP address is high (greater than ninety eight percent), we tend to conjointly claim that the corresponding machine has been confirmed to be compromised. we are able to make sure sixteen of them to be compromised during this means. we tend to note that the bulk ( 62.5 percent) of the IP addresses confirmed by the spam proportion are dynamic IP addresses, that additional indicates the chance of the machines to be compromised.

All the compromised machines are detected with no over eleven observations. this means that, HEURISTIC will quickly discover the compromised machines. we tend to note that HEURISTIC doesn't want compromised machines to send spam messages at a high rate so as to discover them. Here, "quick" detection doesn't mean a brief period, however rather alittle range of observations. A compromised machine will send spam messages at an occasional rate (which, though, works against the interest of spammers), however it will still be detected once enough observations ar obtained by HEURISTIC.

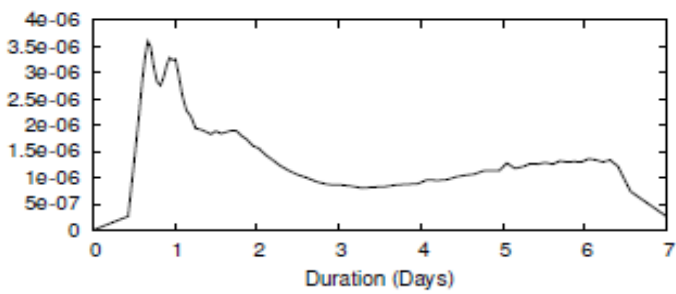

Figure 7.1. Heuristic discover spam zombies in pace 


\section{Conclusion And Future Work}

During this paper, developed an efficient spam zombie detection system named HEURISTIC by observation outgoing messages during a network. In future experiments, build arrange to apply the uniform technique Medicos to the algorithms tested during this work, for obtaining additional comparable results. With relation to the utilization of heuristics, it will see that this data alone isn't competitive, however it will improve classification supported words. the advance shown in our experiments is modest, because of the heuristics used. Here aren't ready to add different heuristics during this case as a result of the Spam base assortment comes during a preprocessed fashion. For future experiments, they're going to use the gathering from that is in raw kind. This reality can modify U.S. to look for additional powerful heuristics.

Additionally, these conjointly showed that HEURISTIC outperforms 2 different detection algorithms supported the amount and proportion of spam messages sent by an enclosed machine, severally.

\section{References}

[1] S. Areora, P. Raghavan, and S. Rao, “Approximation Schemes for Euclidean k-Medians and Related Problems," Proc. 30th ACM Symp. Theory of Computing (STOC), 1998

[2] M. Baker, R. Buyya, and D. Laforenza, "Grids and Grid Technology for Wide-Area Distributed Computing," Software- Practice and Experience, 2002.

[3] A. Chervenak, E. Deelman, I. Foster, L. Guy, W. Hoschek, C. Kesselman, P. Kunszt, M. Ripeanu, B. Schwaretzkopf, H. Stockinger, and B. Tierney, "Giggle: A Framework for Constructing Scalable Replica Location.

[4] F. Li and M.-H. Hsieh. An Empirical Study of Clustering Behavior of Spammers and Group-based Anti-Spam Strategies. In Conference on Email and Anti-Spam, 2006.

[5] Daswani,N., Stoppelman, M., and the google click quality and security teams. The anatomy of clickbot.a. In HotBots'07.

[6] Postini Message Security and Management Update for October Reveals that Spam is Back with a Vengeance. http://postini.com/news events/pr/pr110606.php, 2006.

[7] A. Ramachandran, D. Dagon, and N. Feamster. Can DNSBased Blacklists Keep Up with Bots? In Conference on Email and AntiSpam, 2006.

[8] A. Ramachandran and N. Feamster. Understanding the Network-Level Behavior of Spammers. In Proc. of Sigcomm, 2006.

[9] A. Ramachandran, N. Feamster, and D. Dagon. Revealing Botnet Membership Using DNSBL Counter-Intelligence. In 2nd Steps to Reducing Unwanted Traffic on the Internet Workshop (SRUTI), 2006.

[10] Route Views Project. http://www.routeviews.org.

[15] V. Sekar, Y. Xie, M. K. Reiter, and H. Zhang. A Multi-Resolution Approach for Worm Detection and Containment. In DSN, 2006.

[11] Ramachandran, A., and Feamster, N. Understanding the network-level behavior of spammers. In SIGCOMM'O6. 